\title{
Multiscale Event Study of Private Placement Announcement Effect
}

\author{
Yuling Zhao \\ Experiment Teaching Center \\ Dongbei University of Finance and Economics \\ Dalian, 116023, China \\ E-mail: yulin0415@163.com
}

\begin{abstract}
The importance of understanding the underlying characteristics of private placement Announcement Effect attracts much attention from academic researchers and financial practitioners. Due to the overwhelming complexity of the financial market, many traditional methods such as intervention method and event study fail to produce consistently good analysis results. Empirical Mode Decomposition (EMD), proposed by Huang et al., appears to be a promising data analysis method for nonlinear and nonstationary time series. In this paper, innovative EMD-based multi-scale event analysis method is proposed to estimate the impact of Announcement Date Effect on stock price volatility, and then take illustrative FangDa Group (000055 in Shenzhen Stock Market) to verify the effectiveness of the proposed method. The case study results show that this approach is a promising method from the multi-scale point of view to analyze the impact of Announcement Date Effect in stock market.
\end{abstract}

Keywords-component; Private Placement; empirical mode decomposition; event study;

\section{INTRODUCTION}

Financing has always been an important way for survival and sustainable development of a successful enterprise. It can be divided into two types: debt financing and equity financing. Debt financing has characteristics of due for repayment. When the listed companies need to raise huge amounts of money, often choose equity financing. According to the different channels of financing, equity financing can be divided into a public offering of new shares and the nonpublic issue of new shares (also known as "private placement").

Private placements first emerge in the U.S. market, and flourish in other countries. From 1995 private placement market emerges until 2003 The U.S. private placements grand total of a total of 7594 cases, and raise more than 333.6 billion U.S. dollars, equivalent to $25 \%$ of the funding level of the same period public offering. Since then, the private placement maintains an annual growth rate of more than 10 billion U.S. dollars. The private placement of foreign markets has shown widespread positive announcement effect. Wruck[1] found that in U.S. market from 1979 to 1985, 128 cases of the private placement along with the average excess return of $4.5 \%$. Kato, and Schallheim[2] found that the Japanese stock market reaction of the private placement announcement effect is significantly positive.

Existing literature mainly use event study method to investigative private placement announcement effect. The event study method is a standard analytical tool for assessing the impact of unanticipated event in economics, accounting and financial field [3]. By calculating the normal return of the event window periods, i.e., in the case of the event is based on the normal return model in the estimation window; cumulative abnormal return of the event window periods is estimated, and test whether it is significantly different from zero. The sum of the actual and the predicted return of the difference are defined as the cumulative abnormal returns. It is often regarded as abnormal events caused by the event

In summary, event study method considering the time series as sum of the normal development and a special impact, then they impose a "divide and conquer" strategy respectively to separate mode into two parts. But the solution of Huang [4], a complex time series can be regarded as summary of a few simple oscillating modes. Generally the number of these modes is two or more. In addition, as a special impact, extreme events, may make different type of impulses to these modes. Therefore, a feasible decomposition method which can extract the inherent oscillation from the time sequence It will be more useful to explore extreme events. In this article, we will apply a promising decomposition algorithm, Empirical Mode Decomposition [4], to analyze the events effect, and establish an innovative EMD-based extreme event analysis approach.

In the proposed EMD-based event analysis method, the original time series is broken down into a number of independent local time scale oscillation mainly from high to low, and then the specific implication of each oscillation is identified. Usually, there is a dominant oscillation (or the sum of several oscillations) is solely caused by the extreme event of interest, which is used to approximate the pattern and the change in the amplitude. At the same time, other oscillation provides useful information on different time scales change triggered by events, such events affect the short-term fluctuation or a long-term trend.

An EMD-based method has certain advantages in intervention analysis and the event research methods. First of all, it is suitable for nonlinear and non-stationary data. Intervention analysis and event study method is based on linear models. Second, it provides a multi-scale framework to analyze the impact of Announcement Effect, especially Private Placement Announcement Date Effect and Issue Date Effect, either directly or indirectly. Because it provides a method, from different perspectives, some interesting phenomenon was found. 


\section{EMD-BASED EVENT ANALYSIS}

EMD method is a generally nonlinear, non-stationary data processing method developed by Huang [4]. It considers the real time series as fast oscillations super-imposed on slow oscillations, may have many different modes of oscillations at the same time. EMD can extract the intrinsic modes from original time series, based on the local characteristic scale of data itself, and represent each intrinsic mode as an intrinsic mode function (IMF), that satisfies two conditions:

- The functions have the same numbers of extremes and zero-crossings or differ at the most by one;

- The functions are symmetric with respect to local zero mean.

An extreme event analysis method is formulated based on EMD's performance. The main reasons for choosing EMD method instead of other decomposition methods are as follows:

- EMD is suitable for nonlinear and non-stationary data analysis and the stock prices just have the characteristics of non-stationary and nonlinearity.

- The decomposition process is totally based on the feature of time series, without any a prior basis. For an economic time series, it means any extracted oscillation is actually caused by a concrete factor or event in reality.

The entire process of EMD-based event analysis is described as follows:

\section{A. Data frequency and analysis window determination}

The first step is to select the data according to the event of interest. And then the data is divided into two sub-stages: estimated window and event window. Estimation window is defined as without impact of the events, and the event window is defined as including the impact of events.

The frequency and size of the window of the data limits the time scale of the IMF. According to the Nyquist sampling theorem [5], can be extracted from the signal in the longest period of no more than half of the data points. Therefore, in order to survey the scale of the wide distribution of the data, the high frequency data with a large window size is preferred.

\section{B. EMD decomposition}

After the Preliminary understanding of the events and the identification data, broken down into a number of time-series of the IMF.

\section{Intrinsic modes analysis}

The first task in this step is to find the outline of extreme events by the analysis of the overall change in window mode, the mode. As mentioned earlier, each IMF has a specific meaning and a more significant part of the original time sequence. The effect of extreme events is represented by one or several of sums of the IMF. This IMF or the sum of the IMF treated as the primary mode of treatment in the analysis. Be eliminated due to noise and contain the long-term trend in the original time series, the main mode gives a clear assessment model and the change in the magnitude of extreme events in the event window

Determine the specific meaning of each IMF key is to look at the average cycle, in combination with other techniques, such as graphical comparison of the statistical analysis, fine to coarse reconstruction, spectrum analysis, etc. It should be noted that, if the effect of some of the events included in the different scales superimposed signals, they can be broken down into several IMF. In such a case, these IMF should be grouped into one component.

In addition to the main mode, the event is also likely to affect the other scale time-series analysis. For example, the uncertainties triggered by the events tend to make the analysis of indicators showing rapid small fluctuations. This is done by comparison test of energy (amplitude squared) IMF. Addition, Hilbert spectrum [4] can tell the energy frequency distribution of the difference between the events and the estimated window.

\section{Test of the impact is temporary or permanent}

In order to answer the question whether the effect is temporary, i.e., disappear soon after the event, or permanently to a certain extent, the need for a longer range of data. Only in a long period of time, that can be verified as a real structure of the breakpoint.

\section{E. Economic analysis}

Analysis of the conclusions drawn on the basis, in accordance with steps 1) to 4). Patterns and changes in the size of the event are summarized. Then given the economic interpretation

\section{CASE STUDY}

Two most important dates of stocks: Private Placement Announcement Date (P.P.A Date) and Issue Date are analyzed, in order to illustrate and verify the proposed EMD-based event analysis method. The choosing stock is FangDa Group in China Stock Market; its stock number is 000055. Private Placement Announcement Date is On March 28, 2009. Issue Date is on July 14, 2010

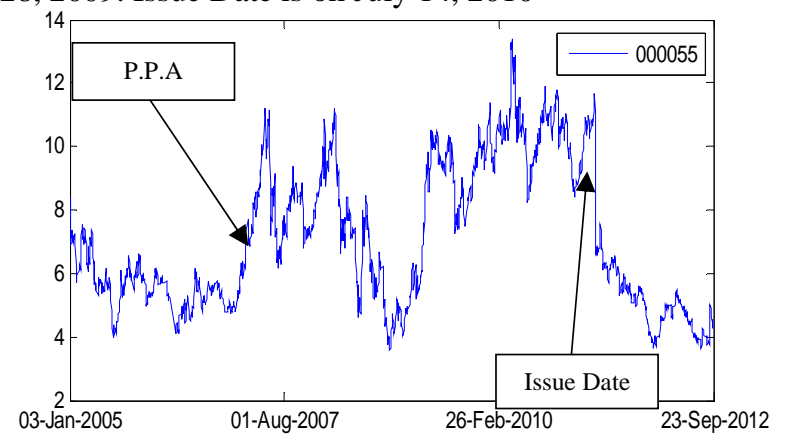

Figure 1. The daily stock prices of 000055 from Jan 3, 2005 to Sep 23, 2012

The impact of the announcement day on stock price is analyzed by five steps proposed in section II. 


\section{A. Data frequency and analysis window determination}

The event window is defined from Private Placement Announcement Date (March 28, 2009) to Issue Date(July 14, 2010) and estimation window is defined as half the span of event window before and after the P.P.A Date from Jan 3, 2005 to Sep 23, 2012.

\section{B. EMD decomposition}

EMD method is applied to decompose the price series (Fig.1) with total of 620 data points. The price series is decomposed into 7 IMF and 1 residue. Fig 2 shows the visualization.

Each component has its own distinct characteristics. The residue is slowly varying around the long term mean. Therefore, it is treated as the long term trend; each sharp up or down of the low frequency component corresponds to a significant event, which should be representative of the effect of these events; the high frequency component, with the characteristics of small amplitudes, contains the effects of stock markets' short term fluctuations.

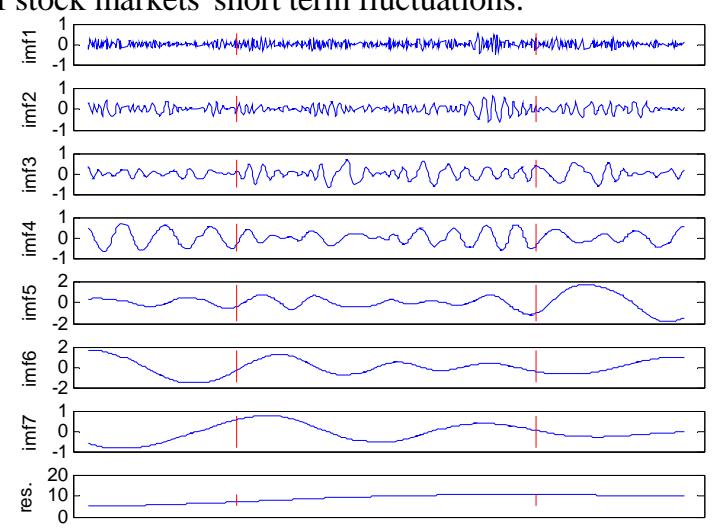

Figure 2. The IMFs and residue for stock price of 000055 from Jan 3,

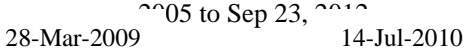

\section{Intrinsic modes analysis}

1) Dominant mode during the event window,

In order to find the dominant mode, we statistical analyze the IMFs. The calculation methods of the three measures are computed: average period, correlation coefficient, and the percentage variance of the original time series. As the frequency and amplitude of the IMF may vary over time, the period is not constant. The average period is defined the value derived by dividing through by the number of the total number of the peak point of each of the IMF. The correlation coefficient was used to measure the various components of the relationship between, IMFs and the original time sequence. Variances of each IMF's percent of the stock series are used to explain the contribution of each IMF observation, because the IMF components are mutually independent.

It is observed that the dominant mode of the prices data is not any IMF but the residue. Both Pearson and Kendall coefficients, between the residue and the observed data, reach a high level of $0.87,0.58$ Tables I. At the same time, variances of the residue account for more than $70.5 \%$ of the total variability. As Huang mentioned [4], the residue is often treated as the deterministic long term behavior. For the longer time price series data, the second important mode is the lowest frequency IMF, IMF7, which has a mean period of nearly 16 months.

TABLE I. MEASURES OF IMFS AND THE RESIDUE FOR STOCK PRICE

\begin{tabular}{|c|c|c|c|c|}
\hline Signal & $\begin{array}{c}\text { Average } \\
\text { Period(day) }\end{array}$ & $\begin{array}{c}\text { Pearson } \\
\text { Correlation }\end{array}$ & $\begin{array}{c}\text { Kendall } \\
\text { correlation }\end{array}$ & $\begin{array}{c}\text { Variance as } \\
\text { \% of prices }\end{array}$ \\
\hline IMF1 & 2.15 & 0.06 & 0.06 & $0.43 \%$ \\
\hline IMF2 & 5.31 & 0.09 & 0.07 & $0.67 \%$ \\
\hline IMF3 & 15.12 & 0.09 & 0.08 & $1.18 \%$ \\
\hline IMF4 & 50.42 & $0.18^{*}$ & $0.14^{*}$ & $2.03 \%$ \\
\hline IMF5 & 66.84 & $0.21^{*}$ & $0.22^{*}$ & $9.53 \%$ \\
\hline IMF6 & 165.37 & $0.24^{*}$ & $0.12^{*}$ & $11.47 \%$ \\
\hline IMF7 & 201.66 & $0.41^{*}$ & $0.22^{*}$ & $3.63 \%$ \\
\hline Residue & & $0.87^{*}$ & $0.58^{*}$ & $70.50 \%$ \\
\hline
\end{tabular}

The sum of variances for the three most important components, the residue and IMF5 IMF6 IMF7, contribute $86.70 \%$ of total variance. On the other hand, the first two IMFs not only exhibit very low correlation coefficients with the observed data but also account for a little more than $1 \%$ of total variance. This means these IMFs do not have serious effect on stock price. So the dominant mode is the sum of IMF5 IMF6 IMF7 and residue.

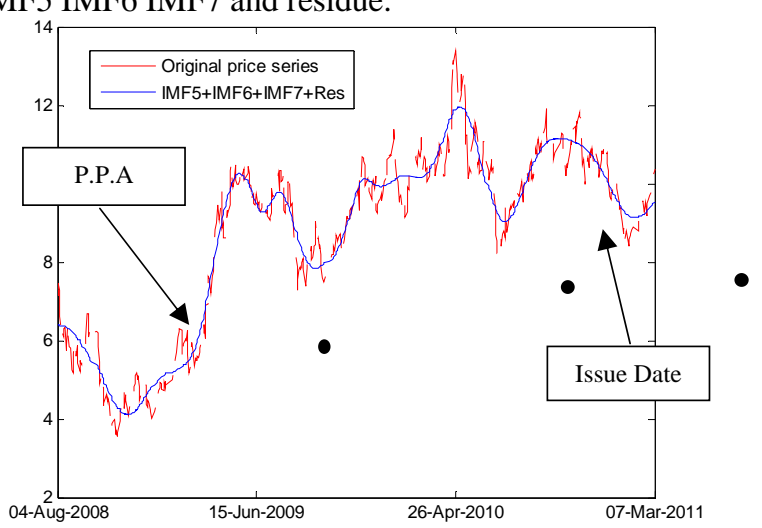

Figure 3. IMF5+IMF6+IMF7+Residue and structure breakpoints

\section{2) The impact of the P.P.A Date and Issue Date effect}

As can be seen from Fig. 3, IMF5 + IMF6 + IMF7 + Residue almost perfectly match the shape of the original time sequence. The first groove in this cycle time is not strictly the same as the P.P.A Date. The source of the differences comes from the EMD algorithm itself. Cubic spline interpolation aims to obtain an extension of the data in the beginning and end of the time series. The final results will appear in the decomposition process, the transmission distance is fine thick IMF the IMF. Furthermore, in the EMD standards, such as stopping, parameters, slight differences may result in extreme IMF position.

However, the distance from the nearest local minimum value and the maximum value is relatively stable, and can be used to measure the amplitude of each cycle. Therefore, in this study, the first to measure the total distance between the local minima and following local maxima invasion of crude oil prices. More specifically, the overall Private Placement 
Announcement impact on price is RMB 2.15 Yuan and Issue Date impact is RMB 1.37 Yuan. By analyzing the dominant mode, instead of original one, the frequency and magnitude of the impact is very clear.

\section{Test of the impact is temporary or permanent}

Breakpoint test method is proposed structure the to test whether there is a breakpoint events around the outbreak [6]. The "strucchange" package in $\mathrm{R}$ software is used to test structure breakpoints within stock price series. The BIC rule show there is three breakpoints which are represented by black dots shown in Fig.3. The closest breakpoint to the Private Placement Announcement Date Effect is Sep 18, 2009 and closest breakpoint to the Issue Date effect is Jan 26, 2011. So both Private Placement Announcement Date and Issue Date have temporary effect that will last 274 days and 196 days.

\section{E. economic analysis}

From the above analysis, some interesting findings about the impact of Announcement Effect can be summarized below:

- Announcement Effect can cause larger vibration as a delta impulse, which is consistent with the time span of the event;

- Small fluctuations are represented by the high frequency of the IMFs have no long-term impact on the stock price. They are caused by daily price fluctuations.

- Announcement Effect on stock prices is like a triangular pulse. This pulse is mainly manifested by the low-frequency IMF and residuals, and accounts for majority of the variations during the event.

\section{CONCLUSION}

In this paper, we first proposed the EMD-based event analysis to estimate the impact of Announcement Date Effect on stock price volatility, and then take illustrative FangDa Group (000055 in Shenzhen Stock Market) to verify the effectiveness of the proposed method. Experimental results show that this approach is promising analysis from the point of view of the multi-scale impact of Announcement Date Effect in stock market.

\section{REFERENCES}

[1] K.H. Wruck, Equity ownership concentration and firm value: Evidence from private equity financings, "Journal of Financial Economics", vol. 23, 1989, pp. 3-28.

[2] K. Kato and J.S. Schallheim, Seasonal and Size Anomalies in the Japanese Stock Market, "The Journal of Financial and Quantitative Analysis", vol. 20, 1985, pp. 243-260.

[3] A.C. Mackinlay, Event studies in economics and finance, "Journal of Economic literature", 1997, pp. 13-39.

[4] N.E. Huang, Z. Shen and S.R. Long, The Empirical Mode Decomposition and the Hilbert Spectrum for Nonlinear and Nonstationary Time Series analysis, "Proceedings of the Royal Society of London", vol. 454, 1998, pp. 903-995.

[5] A.V. Oppenheim and R.W. Schafer, Digital Signal Processing, Englewood Cliffs: Prentice-Hall, 1975.

[6] J. Bai and P. Perron, Computation and analysis of multiple structural change models, "Journal of Applied Econometrics", 2003, pp. 1-22. 